\title{
IOT System To Control The Air Quality In Operating Room
}

\author{
Albert Cahya Yojana ${ }^{a}$ \\ ${ }^{a}$ Master of Informatics Engineering at BINUS, Jakarta, Indonesia, albert.yojana @ binus.ac.id
}

Article History: Received: 10 November 2020; Revised 12 January 2021 Accepted: 27 January 2021; Published online: 5 April 2021

\begin{abstract}
The need for an air quality monitoring system in the operating room that can help users to maintain predetermined quality standards. Air quality parameters that are very important to note are temperature, humidity, and positive pressure. The results of the research show that this system can help users to control the air quality system in the operating room, and can provide an alarm in the form of WA to users, with $95.7 \%$ successful delivery. The reading results have been compared with a calibrated instrument. With an average temperature difference of $0.24 \%, \mathrm{RH} 0.18 \%$, DP $0.23 \%$. The purpose of this paper is to develop a control system in the operating room, so that the parameters that become the main standards can be achieved. If any parameter exceeds the predetermined limit, the system will provide a warning or notification in the form of WA to the user.
\end{abstract}

Keywords: Indoor air quality, IOT, operating room

\section{Introduction}

SSI is one of the most common causes of serious surgical complications (Kim, Chu and Shin, 2014) (Surgery and Lives, 2009). SSI accounts for $14 \%$ to $17 \%$ of all hospital illnesses, and $38 \%$ of hospital-related infections come from surgical patients (Weigelt et al., 2007) ('National Nosocomial Infections Surveillance (NNIS) System Report , Data Summary from January 1992-June 2001, Issued August 2001', no date). Each SSI patient will get additional surgery about 7-10 days after the initial operation. Surgical patients with SSI have a 2-11 times higher risk of death, compared to surgical patients without SSI (Engemann et al., 2003) (Anderson et al., 2012).

There are several parameters to maintain good indoor air quality. The temperature must be conditioned so that people in the room feel comfortable. Humidity must also be conditioned, so that fungus or bacteria do not grow. Positive pressure in the operating room or differential pressure between the operating room and the corridor, must also be considered so that dirty air that has not been filtered does not enter the room. Many users do not close the door tightly so that positive pressure in the room is not reached or reduced. Thus it can cause the entry of dirty air from outside the operating theatres (Roth et al., 2019). Previous tests that have been carried out have concluded positive pressure and door conditions greatly affect the dust that can enter the operating theaters (Teter et al., 2017). Room airflow must remain stable so that the flow of wind that enters the room remains laminar so that the air in the room will quickly be pulled back into the AHU (Air Handling Unit) to filter again (McHugh, Hill and Humphreys, 2015).

In recent months the level of air quality in Jakarta is very bad. Jakarta gets the first rank of the worst air in the world. It will be very dangerous in the long run. some sterile rooms such as operating theatres, food production rooms, injection drug production rooms, and special rooms. Poor air quality in the operating theatres is very dangerous because it can cause SSI to patients. In the operating theatres need air with good air quality. Poor air quality also has an impact on the production space which can cause product failure or not be included in the quality of the product to be produced. Many parameters must be set when the air quality deteriorates.

The control system with the Internet of Things (IOT) can help the user to be set automatically, and the user only has to monitor the results of the control that has been set automatically. Internet of Things (IoT) is one of the results of technological developments that are still evolving. The development of technology itself is increasingly rapid with the support of the Internet and several other components that are also developing. IoT itself is a system or idea or concept where certain objects can transfer data through the Internet without the need for human assistance to transfer the data. With the concept of IoT, it can access and read the status of the sensor wherever the user is, by using an Internet connection (Kodali et al., 2017). Because with the IoT system, the sensor will work and educate itself without human assistance anymore (Wang et al., 2006).

Operating room air is conditioned by an air handling unit (AHU). AHU gets cold water through the chiller. AHU fans will draw wind from the room and outside air, the wind will be filtered with a pre-filter and filter media. After that, it will pass the cooling coil which is already very cold because of the water chiller, so that air 
can condense as it passes through the cooling coil. Then, the air will pass through the heater so that the temperature can be returned to the desired temperature. After that, the wind will be blown into the room but through the last filter, the HEPA filter. As shown in "Fig 1".

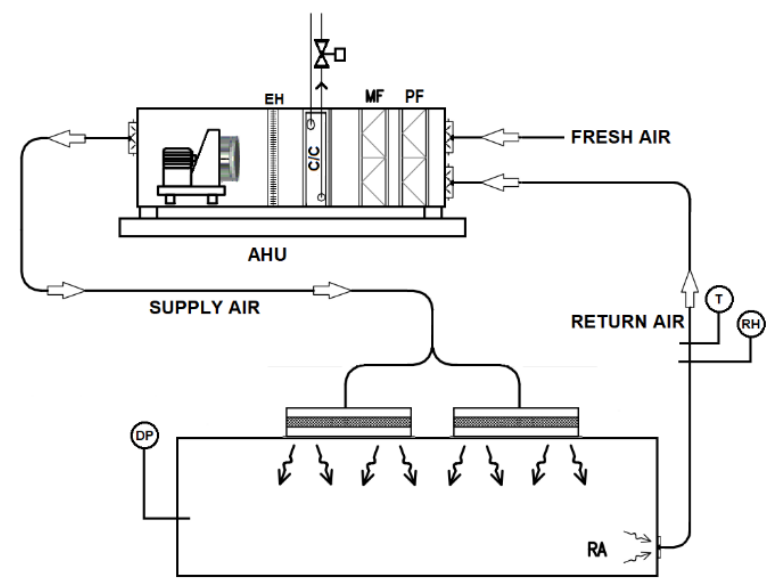

Fig.1. System operating theaters

\section{Related Works}

Research conducted by Brienza (Brienza et al., 2015), using a simple and inexpensive tool. Research is used to monitor gas in several areas. Liberal sensors are placed in several points in the area, each sensor will send data to the server. So that the user can read / see it through the application. The drawback is that users can only see it through a new smartphone. The system does not have an alarm if the gas in the sensor area exceeds the normal limit.

Research conducted by Fiocolla (Fioccola et al., 2016), researchers use Arduino and cloud systems to manage data obtained through air quality sensors (AQ). Researchers compared 2 communication services for IOT, namely Rest API and Message Queuing Telemetry Transport (MQTT). Researchers are still using cables so that the mobility is less good, and the alarm system does not exist when the AQ sensor exceeds the normal limit.

Research conducted by Zheng (Zheng et al., 2016), the research that has been done here is to monitor the air quality in the city. The sensor used is pm2.5, temperature, and humidity sensor. The data will be sent to the server so that the server can process the data and convert it into a graph for analysis. In this study, there is still no alarm that can tell if the sensor reading is outside the safe limit.

Research conducted by Kumar (Phala, Kumar and Hancke, 2016), The researchers monitored the air quality system according to IEEE / ISO / IEC 2145 standards. The research used CO, CO2, SO2 and NO2 sensors, all sensor data readings were sent to the server. The drawback of this research is that it does not have an alarm if a reading is outside the standard limit. Research conducted by Tsang (Tsang et al., 2016), This research uses IAQM as an object to test the WSN zigbee which is more energy efficient and better. but research is not applied in the field, only in the testing room. Researchers used OPNET as software for testing the latency of the WSN zigbee communication.

Research conducted by Firdous (Firdous, Sudantha and Karunaratne, 2017), research conducted using the O3 sensor to measure the gas levels in a room. The $\mathrm{O} 3$ sensor is placed in the room because of the photocopier in it. The data from the sensor uses bluetooth and is processed on the server using wifi. The system that is designed does not have an alarm if the $\mathrm{O} 3$ level in the room is high. Research conducted by Marinov (Marinov et al., 2016), research conducted using amperometric sensors and gas sensors $(\mathrm{O} 2, \mathrm{O} 3, \mathrm{CO}$, and $\mathrm{CO} 2)$ were controlled using the PIC18F87K22 as a microcontroller. Sensors are placed at several points, to send data to the server. The data obtained will be displayed on a map.

Research by Kumar (Kumar and Jasuja, 2017), this study uses several types of sensors. Researchers used six sensors as a tool to retrieve IAQM data. The installed sensor is connected to Arduino Uno and Raspberry PI, then using WIFI to send data to the cloud, so that the data can be seen by users with their devices. Researchers use the IBM Watson Platform as an interface display. The system used does not have a graphical report in which the data have seen only displays real-time readings at that time. Research by Benammar (Benammar et al., 2018), researchers used a fairly complete sensor module. There are six sensors installed directly connected to one board. Researchers use a 6,600 mAH power bank as backup power when the power is off. So that communication will be 
more awake. Researchers use Raspberry PI2 and Xbee-ZB controllers to send data to the server. The system created already uses graphics so that users are easier to see. But no warning is given if the parameter exceeds the limit.

\section{Research Framework/Methodology}

As shown in "Fig 2". the system to be made is by using temperature sensors, humidity sensors, differential/delta pressure sensors, and carbon monoxide. These main sensors are part of the system to be made. The operating room will be measured by a sensor and connected to a micro-controller (ESP32). ESP32 functions as a micro-controller that will read sensor data and process the data to control solenoid 2 -way valve and heater step.

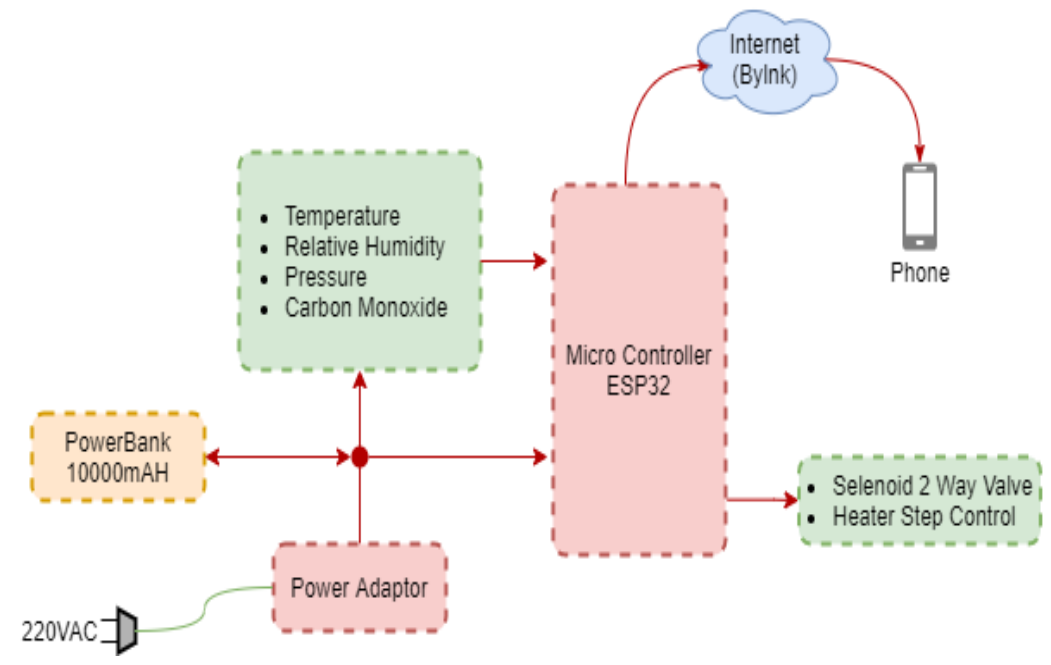

Fig.2. System configuration IAQM

\section{Proposed Methods}

The designed control system can be seen in Fig. 3 and Fig. 4. Data sensors that have been read are sent to the internet and can be viewed on mobile phones with the Bylnk application. Sensor data can also be viewed in graphical form in the Bylnk application. As shown in "Fig 3". when system start will be intialization the parameter temperature, RH, Differential Pressure (DP), and Carbon Monoxide (CO). System will read sensor temperature data. If the temperature is more than $22^{\circ} \mathrm{C}$ the heater will turn off, if the temperature is below $22^{\circ} \mathrm{C}$ then the heater will be on. System will also be managed to provide warnings to operators in the form of WA. A warning will be given if the temperature exceeds $24^{\circ} \mathrm{C}$ for 1 minute. System will read the data from humidity sensor. If humidity more than $53 \%$ solenoid 2 -way valve will open, if humidity is less than $53 \%$ then solenoid 2 way valve will close. A warning will also be given if the humidity exceeds $60 \%$ for 3 minutes. 


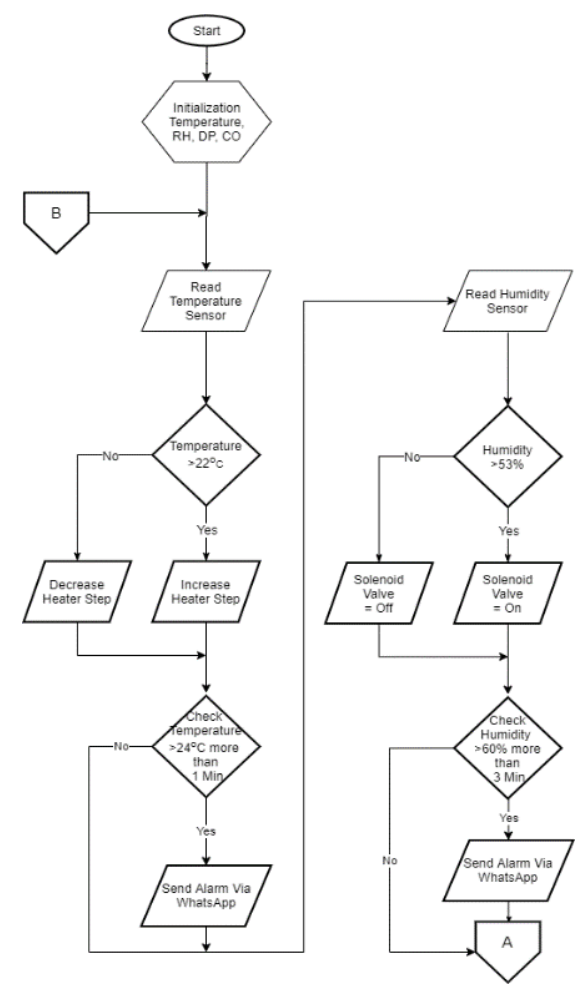

Fig.3. Flowchart system diagram temperature and humidity

As shown in "Fig 4". system will be read differential pressure sensor and system will be give some warning also if the DP is less than 5 Pascal or more than 20 Pascal for more than 1 minute. System will be read carbon monoxide sensor and give warning, if carbon monoxide is more than 10 micrograms per cubic meter $(\mu \mathrm{g} / \mathrm{m} 3)$ for more than 5 minutes.

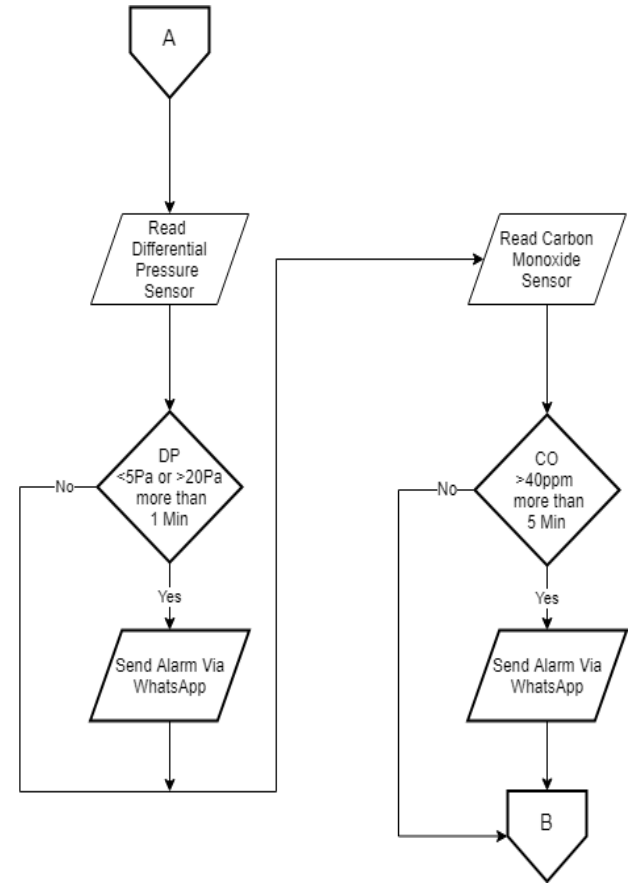

Fig.4. Flowchart system diagram differential pressure and carbon monoxide

All sensor data will be accessed or viewed via mobile phones using the bylnk application. Actual temperature, humidity, differential pressure and carbon monoxide readings can be seen in the application. Display charts in 
chart form and change the setpoint for temperature and humidity can be done in the application. Overview shown in "Fig 5". green for temperature readings, purple for humidity readings, blue for differential pressure readings, and orange for carbon monoxide sensor readings. A small historical chart is visible below, and for setting the temperature and humidity are also available.

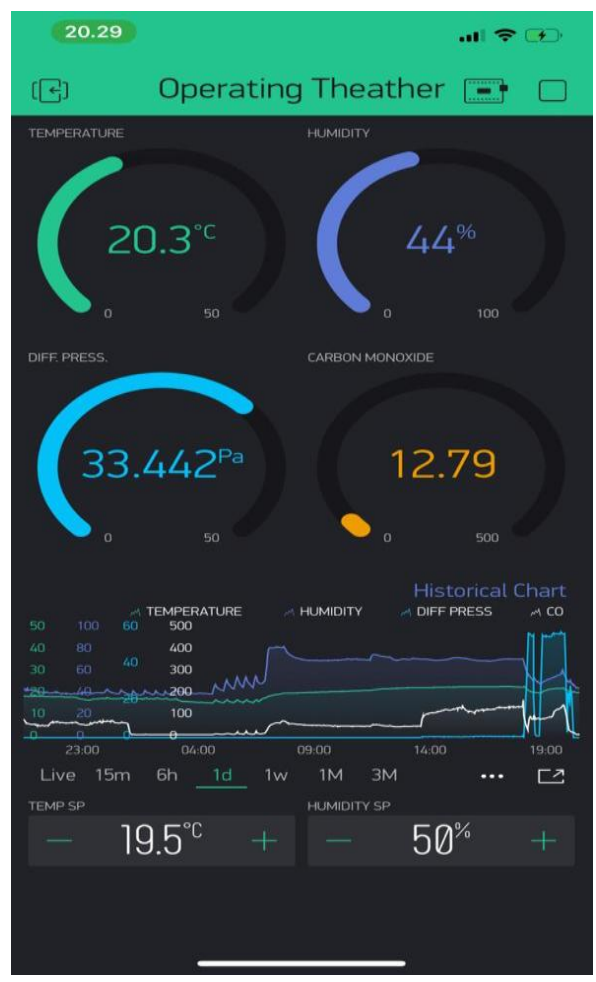

Fig.5. Display overview in mobile phone

As shown in "Fig 6". historical full screen view of the chart is also available. so that the user can easily analyze when temperatures start to rise, or when parameters start out of the limits.

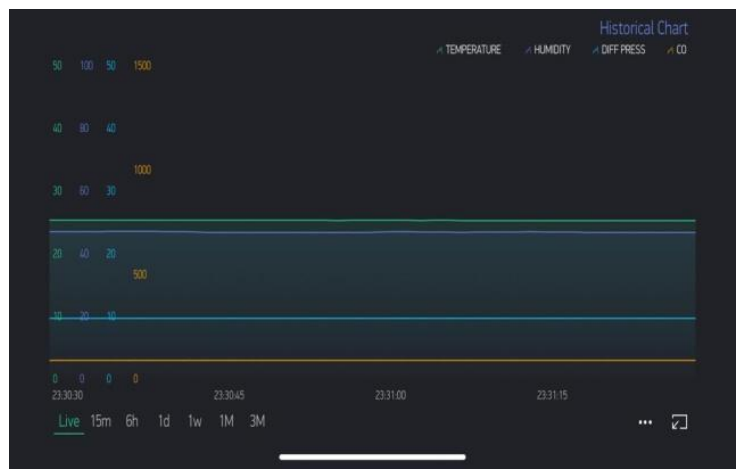

Fig.6. Fullscreen historical chart in mobile phone

Heater is designed to have 3 steps. Relay 1 has a capacity of 2,000 watts, relay 2 has a capacity of 4,000 watts, relay 3 has a capacity of 8,000 watts. Each step is made different and increases 2x compared to the previous step. This is so that controlled air can be more stable. When compared with a heater with 1 step, the one with more steps is better. By using 3 relay contacts with binary steps, it can produce 7 steps, as shown in Table 3.1. When the air is not hot enough, the program will raise the heater using binary steps. So that the control obtained is more stable compared to the usual step which uses 3 relays too. 
Table 1. Binary Step Heater

\begin{tabular}{|l|l|l|l|l|}
\hline Step & Relay 3 & Relay 2 & Relay 1 & Total Watt \\
\hline 1 & 0 & 0 & 1 & 2.000 Watt \\
\hline 2 & 0 & 1 & 0 & 4.000 Watt \\
\hline 3 & 0 & 1 & 1 & 6.000 Watt \\
\hline 4 & 1 & 0 & 0 & 8.000 Watt \\
\hline 5 & 1 & 0 & 1 & 10.000 Watt \\
\hline 6 & 1 & 1 & 0 & 12.000 Watt \\
\hline 7 & 1 & 1 & 1 & 14.000 Watt \\
\hline
\end{tabular}

The circuit is intended to connect the ESP32 and the sensor used. So that the ESP32 as a control module can read the sensors that are used properly. The controller circuit can be seen in "Fig 7". S1 is a temperature and RH sensor, has 3 pins, pin + gets $5 \mathrm{~V}$, pin - gets GND, and pin out gets TRH. S2 is a CO sensor, has 4 pins, VCC pin gets $5 \mathrm{~V}$, GND pin gets GND, and pin AO gets AO. S3 is a mini display that can display sensor readings, has 4 pins, VCC pins get 5V, GND pins get GND, SCL pins get SCL, and SDA pins get SDA. S4 is a relay to command the solenoid valve and heater step, has 6 pins, VCC pins get 5V, GND pins get GND, IN1 pins get RLY1, IN2 pins get RLY2, IN3 pins get RLY3, and IN4 pins get RLY4.TR is a transformer that aims to change the PLN voltage. From 220VAC to 24VAC. 24VAC is required by the PDT12 sensor as a power supply for the sensor. S5 is PDT12 which functions to read the difference in air pressure, has 4 terminals, terminal 1 gets $24 \mathrm{VAC}$, terminal 2 gets $0 \mathrm{~V}$, terminal 3 is given a resistance of $1 \mathrm{~K} \Omega$ before getting $\mathrm{VO}$, and terminal 4 is given a resistance of $2.7 \mathrm{~K} \Omega$ before getting GND, this aims so that the voltage received by the ESP32 is not more than $3.3 \mathrm{~V}$.

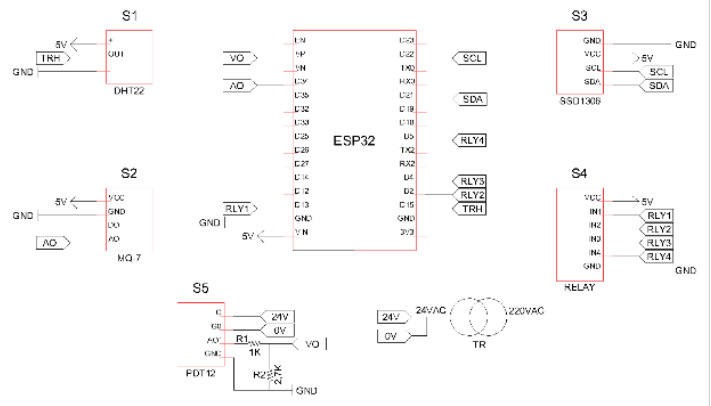

Fig.7. Wiring diagram

\section{Conclusion}

Based on the results of the research that has been done, it can be concluded:

1. The results of the research show that this system can help users to control the air quality system in the operating room. With an average difference in temperature reading of $0.24 \%$, RH $0.18 \%$, and DP $0.23 \%$ with a calibrated tool.

2. Users can find out more quickly, when a parameter is out of the specified limit.

3. Users can monitor parameters from anywhere, with an internet connection.

So that from this research, the researcher can control the parameters that are set at predetermined limits. If there is a problem with the predetermined parameters, an alarm will be sent via WA to the user. With this IoT system, users do not need to be on standby at all times. Because, all parameters can be seen via a smartphone with an internet connection.

\section{References}

Anderson, D. J. et al., "Strategies to Prevent Surgical Site Infections in Acute Care Hospitals", doi: 10.1086/591064, 2012.

Benammar, M. et al., "A modular IoT platform for real-time indoor air quality monitoring", Sensors (Switzerland), 18(2), pp. 1-18, doi: 10.3390/s18020581, 2018. 
Brienza, S. et al., "A low-cost sensing system for cooperative air quality monitoring in urban areas", Sensors (Switzerland), 15(6), pp. 12242-12259, doi: 10.3390/s150612242, 2015.

Engemann, J. J. et al., "Adverse Clinical and Economic Outcomes Attributable to Methicillin Resistance among Patients with Staphylococcus aureus Surgical Site Infection", 27710, 2003.

Fioccola, G. B. et al., "Polluino: An efficient cloud-based management of IoT devices for air quality monitoring", 2016 IEEE 2nd International Forum on Research and Technologies for Society and Industry Leveraging a Better Tomorrow, RTSI 2016. doi: 10.1109/RTSI.2016.7740617, 2016.

Firdous, M. F. M., Sudantha, B. H. and Karunaratne, P. M., "IoT Enabled Proactive Indoor Air Quality Monitoring System for Sustainable Health Management", pp. 216-221, 2017.

Kim, J. Y., Chu, C. H. and Shin, S. M., "ISSAQ: An integrated sensing systems for real-time indoor air quality monitoring", IEEE Sensors Journal, 14(12), pp. 4230-4244. doi: 10.1109/JSEN.2014.2359832, 2014.

Kodali, R. K. et al., "IoT based smart security and home automation system", Proceeding - IEEE International Conference on Computing, Communication and Automation, ICCCA 2016, pp. 1286-1289. doi: 10.1109/CCAA.2016.7813916, 2017.

Kumar, S. and Jasuja, A., "Air quality monitoring system based on IoT using Raspberry Pi", Proceeding - IEEE International Conference on Computing, Communication and Automation, ICCCA 2017, 2017-Janua, pp. 1341-1346. doi: 10.1109/CCAA.2017.8230005, 2017.

Marinov, M. B. et al., "Air Quality Monitoring in Urban Environments", pp. 443-448, 2016.

McHugh, S. M., Hill, A. D. K. and Humphreys, H., "Laminar airflow and the prevention of surgical site infection. More harm than good?", Surgeon. Elsevier Ltd, 13(1), pp. 52-58. doi: 10.1016/j.surge.2014.10.003, 2015.

National Nosocomial Infections Surveillance ( NNIS ) System Report, Data Summary from January 1992-June 2001 , Issued August 2001' (no date), (August 2001), pp. 404-421. doi: 10.1067/mic.2001.119952.

Phala, K. S. E., Kumar, A. and Hancke, G. P., "Air quality monitoring system based on ISO/IEC/IEEE 21451 standards", IEEE Sensors Journal, 16(12), pp. 5037-5045. doi: 10.1109/JSEN.2016.2555935, 2016.

Roth, J. A. et al., "Frequent Door Openings During Cardiac Surgery Are Associated With Increased Risk for Surgical Site Infection: A Prospective Observational Study", Clinical Infectious Diseases, 69(2), pp. $290-294$. doi: 10.1093/cid/ciy879, 2019.

Surgery, S. and Lives, S., "WHO Guidelines for Safe Surgery 2009", 2009.

Teter, J. et al., "Assessment of operating room airflow using air particle counts and direct observation of door openings", American Journal of Infection Control. Elsevier Inc., 45(5), pp. 477-482. doi: 10.1016/j.ajic.2016.12.018, 2017.

Tsang, K. F. et al., "Energy-saving IAQ monitoring ZigBee network using VIKOR decision making method", Proceedings of the IEEE International Conference on Industrial Technology, 2016-May, pp. 2004-2009. doi: 10.1109/ICIT.2016.7475075, 2016.

Wang, M. et al., "An IoT-based Appliance Control System for Smart Homes", pp. 744-747. Available at: http://lib.gen.in/ocean/e0b70e304d6b05b8e5413d79fc4e4ab4/wang2013.pdf, 2006.

Weigelt, J. A. et al., "Surgical site infections : Causative pathogens and associated outcomes", American Journal of Infection Control. Elsevier Ltd, 38(2), pp. 112-120. doi: 10.1016/j.ajic.2009.06.010, 2007.

Zheng, K. et al., "Design and Implementation of LPWA-Based Air Quality Monitoring System", IEEE Access, 4, pp. 3238-3245. doi: 10.1109/ACCESS.2016.2582153, 2016. 